\title{
- Case Reports-
}

\section{Conservative Management of Hyperreactio Luteinalis: A Case Report}

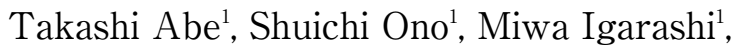 \\ Shigeo Akira ${ }^{1}$, Atsushi Watanabe ${ }^{2}$ and Toshiyuki Takeshita ${ }^{1}$ \\ ${ }^{1}$ Division of Reproductive Medicine, Perinatology and Gynecologic Oncology, Graduate School of Medicine, Nippon Medical School \\ ${ }^{2}$ Division of Clinical Genetics, Nippon Medical School
}

\begin{abstract}
Hyperreactio luteinalis (HL) is characterized by bilaterally enlarged ovaries containing multiple self-limited theca lutein cysts and is usually associated with increased production of human chorionic gonadotropin in the presence of trophoblastic disease or multiple pregnancies. About 30 cases of HL have been reported in patients with normal singleton pregnancies, and most of these patients required surgical intervention during their first pregnancy because of ovarian torsion or to rule out of ovarian malignancy. However, the accurate diagnosis of HL by means of laboratory tests and magnetic resonance imaging and the exclusion of ovarian malignancy in asymptomatic pregnant woman may avoid unnecessary surgical intervention or termination of pregnancy. The present report describes case of HL in a woman with a normal singleton pregnancy in whom conservative management allowed the preservation of both ovaries.
\end{abstract}

(J Nippon Med Sch 2011; 78: 241-245)

Key words: OHSS, FSH receptor, hyperandrogenism, pregnancy

\section{Introduction}

Hyperreactio luteinalis (HL) is a rare condition during spontaneous pregnancy and is characterized by multiple cysts and bilateral ovarian enlargement associated with high maternal serum levels of human chorionic gonadotropin (hCG) and a hyperandrogenic state ${ }^{1-3}$.

Ovarian hyperstimulation syndrome (OHSS) is a well-known complication of the early first trimester of pregnancy and is usually a consequence of iatrogenic ovarian induction, but in rare occasions it can occur spontaneously, particularly in patients with hypothyroidism, a pituitary gonadotropin (follicle-stimulating hormone [FSH]/luteinizing hormone [LH])-secreting adenoma, polycystic ovary syndrome (PCOS), or mutation of the $\mathrm{FSH} / \mathrm{LH}$ receptor ${ }^{4,5}$.

It may be difficult to differentiate severe HL in the first trimester from spontaneous OHSS, but HL may be asymptomatic and only discovered incidentally during routine pelvic ultrasonography or cesarean section.

We recently treated a patient with bilateral ovarian enlargement during the first trimester of pregnancy in whom HL was diagnosed and managed conservatively.

Correspondence to Takashi Abe, MD, PhD, Department of Obstetrics and Gynecology, Nippon Medical School, 1-1-5

Sendagi, Bunkyo-ku, Tokyo 113-8603, Japan

E-mail: takashi360@nms.ac.jp

Journal Website (http://www.nms.ac.jp/jnms/) 


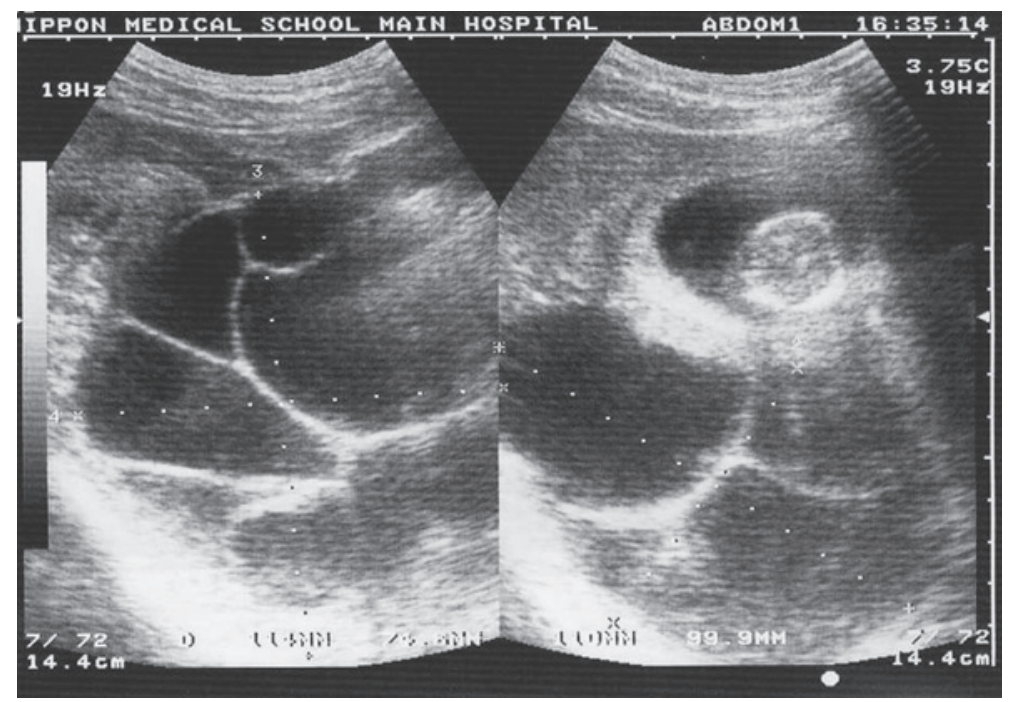

Fig. 1 Transvaginal ultrasonography showed bilateral enlargement of the ovaries (left ovary, $14 \times 11 \mathrm{~cm}$; right ovary, $14 \times 9.9 \mathrm{~cm}$ ) with multiple anechoic cysts.
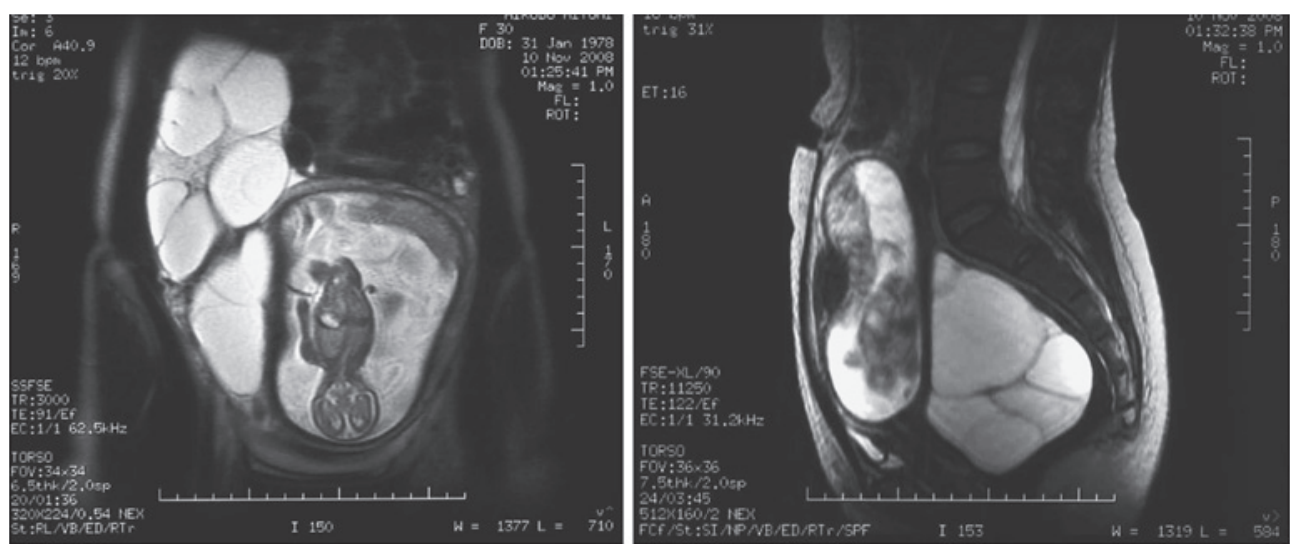

Fig. 2 MRI revealed multiple cystic ovaries (left ovary, $14 \times 10 \mathrm{~cm}$; right ovary, $15 \times 10 \mathrm{~cm}$ ).

\section{Case Report}

A 31-year-old primigravida was referred to our department in the 12 th week of pregnancy. The patient had never taken drugs to induce ovulation. Transvaginal ultrasonography showed a normal singleton pregnancy at 12 weeks' gestation and bilateral enlargement of the ovaries (left ovary, $14 \times$ $11 \mathrm{~cm}$; right ovary, $14 \times 9.9 \mathrm{~cm})$ with multiple anechoic cysts (Fig. 1).

Laboratory results at 12 weeks' gestation included: hematocrit, 39.6\% (normal: 33\%-43\%), liver enzymes and coagulation profiles were normal; blood urea nitrogen, creatinine, and electrolytes were within normal limits; hCG, 104,963 IU/mL; estradiol, 9,599 $\mathrm{pg} / \mathrm{mL}$; progesterone, $48.2 \mathrm{ng} / \mathrm{mL} ; \mathrm{FSH},<1.0 \mathrm{mIU} /$ $\mathrm{mL}$; LH, <0.2 mIU/mL; CA125, $23.9 \mathrm{IU} / \mathrm{mL}$ (normal, $<35$ ); CA19-9, <1.2 (normal, <35); thyroid stimulating hormone, $0.222 \mathrm{IU} / \mathrm{mL}$ (normal, <5.00); free thyroxine 4, $\quad 1.43 \mathrm{ng} / \mathrm{mL}$ (normal, 0.83-1.64, ng/mL); and prolactin, $75.9 \mathrm{ng} / \mathrm{mL}$ (normal, $1.4-14.6 \mathrm{ng} / \mathrm{mL}$ ). Androgen levels were significantly increased: testosterone, $324 \mu \mathrm{g} / \mathrm{mL}$ (normal, $60-150 \mu \mathrm{g} / \mathrm{mL}$ ); free testosterone, $2.2 \mathrm{pg} / \mathrm{mL}$, (normal, $<1.9 \mathrm{pg} / \mathrm{mL}$ ); and androstenedione; $24 \mathrm{ng} / \mathrm{mL}$ (normal, 0.9-3.5 ng/ $\mathrm{mL}$ ). Possible interventions, such as laparotomy, were discussed with the patient and her husband. On the basis of previous reports of HL, we selected conservative management. Magnetic resonance imaging (MRI) was performed to rule out ovarian malignancy (Fig. 2). 
At 38 week's gestation, a healthy female neonate (weight, 2,608 g; Apgar score, 8-9) was delivered by means of cesarean section. Because of a swollen right ovary on the Douglas pouch, cesarean section was performed on the basis of a diagnosis of disturbance of the birth canal.

Intraoperative inspection of the abdomen revealed that the ovaries were grossly enlarged and polycystic (Fig. 3). The mean diameter of the right ovary was $12 \mathrm{~cm}$ and that of the left ovary was 15 $\mathrm{cm}$, with multiple cysts 3 to $4 \mathrm{~cm}$ in diameter and filled with yellowish fluid. Samples of the cystic fluid and specimens from wedge resection of both ovaries and the placenta were submitted for pathologic examination. The abdomen was explored, and no other abnormalities were found.

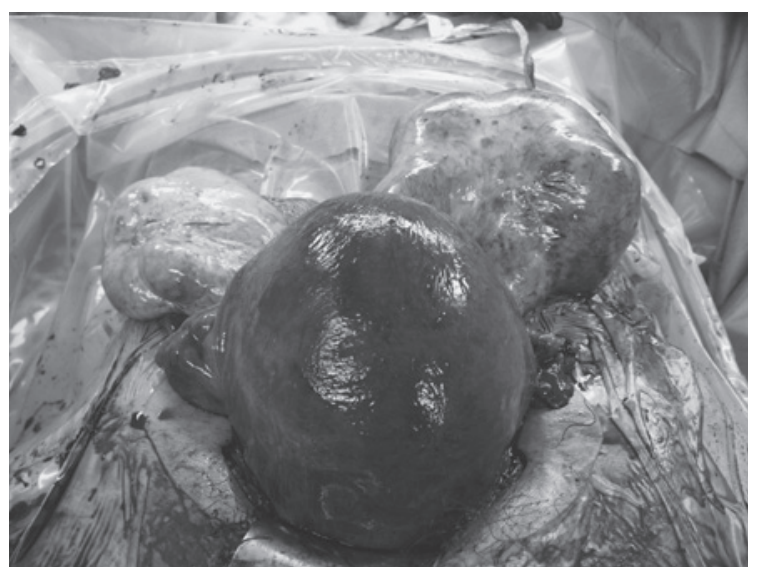

Fig. 3 Both ovaries appeared grossly enlarged and polycystic.
The patient's postoperative course was unremarkable. Ovarian volume decreased slowly and became normal in 4 months. Androgen levels decreased slowly and became normal in 3 months. The female neonate showed no signs of virilization. The pathological diagnosis was hyperreactio luteinalis. The cyst lining comprised granulosa and theca layers, both of which exhibited marked luteinization (Fig. 4).

Sequencing analysis of the gene for the FSH receptor was approved by the ethics committee of Nippon Medical School and was performed with the cooperation of Sabine Costagliola, Ph.D., of Brussels, Belgium, after the patient had given written informed consent. However, the gene for the FSH receptor was normal.

\section{Discussion}

$\mathrm{HL}$ is a rare benign condition, and about 30 cases of HL in normal singleton pregnancies have been reported. Most of the patients required surgical intervention during their first pregnancy because of ovarian torsion or to rule out of ovarian malignancy.

HL is typically a self-limited syndrome with asymptomatic bilateral enlargement of the ovaries containing multiple theca lutein cysts due to excessive hCG stimulation of the ovaries ${ }^{6}$. The hCG is produced by the trophoblast in the first trimester, and its highest median level is $100,000 \mathrm{IU} / \mathrm{L}$ in the

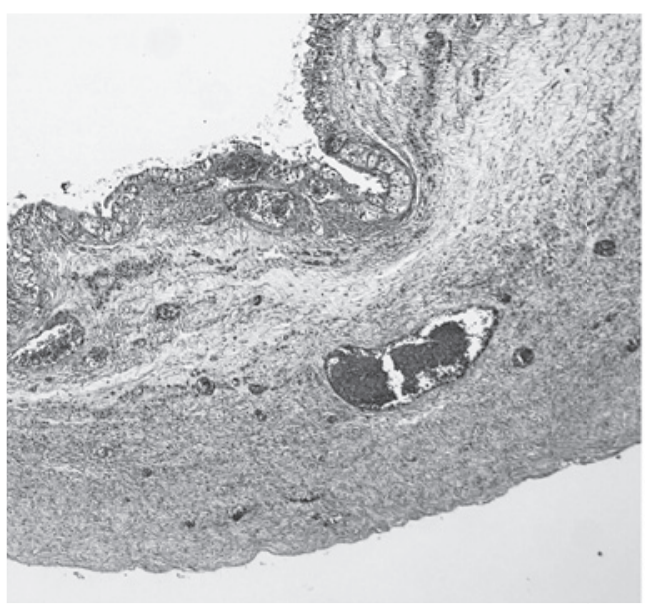

$\mathrm{HE} \times 4$

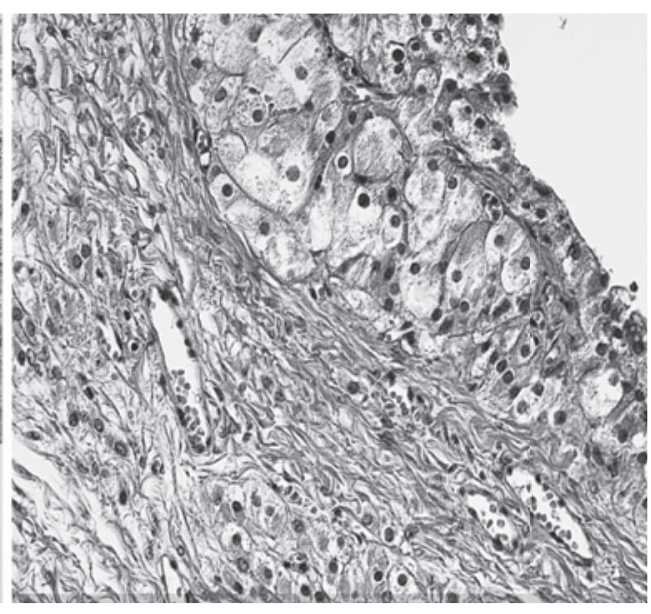

$\mathrm{HE} \times 40$

Fig. 4 The cyst lining comprised granulosa and theca layers, both of which exhibit marked luteinization. 
9th week of gestation ${ }^{6}$. Abnormally high levels of hCG can be found in molar and multiple pregnancies and in specific ovarian or gestational malignancies, fetal chromosomal abnormality, and $\mathrm{HL}^{6}$. On the other hand, the onset of HL is variable ${ }^{7-9}$, and its relation to the level of $\mathrm{hCG}$ is inconsistent $\mathrm{t}^{7.10}$. In the present case, levels of hCG were not abnormally elevated.

It is important to differentiate HL from malignant ovarian tumors to avoid unnecessary surgery. The laboratory findings of high levels of hCG, hyperandrogenism, and normal levels of ovarian tumor markers, in combination with such imaging methods as ultrasonography and MRI, help differentiate HL from malignant ovarian masses.

Bilateral multicystic ovarian tumors may also mimic spontaneous OHSS, but pregnant woman with OHSS usually have a history of induction of ovulation. Also, OHSS has been demonstrated to develop with hypothyroidism, PCOS, FSH-secreting adenoma, or mutation of the FSH receptor ${ }^{4}$. Smits et $\mathrm{al}^{11}$ have found that a mutation of the FSH receptor causes hypersensitivity to hCG which can mimic pharmacologically induced OHSS. Our patient had no history of ovulation induction or therapy for PCOS and showed no hypothyroidism, increase in FSH levels, or mutation of the FSH receptor. Therefore, on the basis of these findings, it is still difficult to differentiate severe HL from spontaneous OHSS because the causes of HL and spontaneous OHSS remain unknown.

The diagnosis of HL may be established at any time during gestation. The majority cases (54\%) are diagnosed in the third trimester or in the puerperium and present with bilateral enlargement of the ovaries and without other symptoms, whereas $16 \%$ are diagnosed in the first trimester ${ }^{12}$. More than $37 \%$ of cases are diagnosed at the time of cesarean section, because HL is asymptomatic in most patients. In contrast, OHSS presents at the beginning of the first trimester and is associated with more severe symptoms involving acute fluid imbalances (such as massive ascites and hemoconcentration) that impair the natural course of pregnancy. The present case was followed up from the first trimester until delivery.
Symptomatic patients present abdominal discomfort or pain, which is a result of ovarian torsion. HL can be associated with hyperemesis gravidarum, hyperthyroidism, hyperandrogenism, intrauterine growth restriction (IUGR), eclampsia, or hemolysis, elevated liver enzymes, and low platelet count (HELLP) syndrome ${ }^{13-16}$.

Hyperandrogenism is a characteristic of $\mathrm{HL}$, but patients with hyperemesis gravidarum and PCOS also show elevated androgen levels. Hormonal studies in the present case confirmed hyperandogenemia; thus, maternal virilization was not seen.

The elevated levels of hCG produced by the trophoblast in the first trimester in an otherwise normal pregnancy could be a sign of poor or incomplete placental invasion ${ }^{13,14}$. The poor placental invasion could lead to preeclampsia, severe IUGR, or HELLP syndrome ${ }^{13-16}$. Because the hCG levels were normal, the present patient was asymptomatic.

In conclusion, we have described a patient with HL presenting as bilateral ovarian enlargement during the first trimester of pregnancy in which conservative management allowed preservation of both ovaries. Although HL is extremely rare in normal singleton pregnancies, it is important to differentiate HL from malignancies to avoid unnecessary surgery.

Acknowledgements: The authors thank Sabine Costagliola, Ph.D., Institut de Recherche Interdisciplinaire en Biologie Humaine et Moleculaire, Brussels, Belgium, for sequencing the FSH receptor gene.

\section{References}

1. Bradshaw KD, Santos-Ramos R, Rawlins SC, MacDonald PC, Parker CR Jr: Endocrine studies in a pregnancy complicated by ovarian theca lutein cysts and hyperreactio luteinalis. Obstet Gynecol 1986; 67: 66S-69S.

2. Muechler EK, Ficher J, Zongrone J: Human chorionic gonadotropin, estriol, and testosterone changes in two pregnancies with hyperreactio luteinalis. Am J Obstet Gynecol 1987; 157: 1126-1128.

3. Takeda T, Minekawa R, Makino M, Sugiyama T, Murata Y, Suehara N: Hyperreactio luteinalis associated with severe twin-to-twin transfusion syndrome. Gynecol Obstet Invest 2002; 53: 243-246.

4. Foulk RA, Martin MC, Jerkins GL, Laros RK: 
Hyperreactio luteinalis differentiated from severe ovarian hyperstimulation syndrome in a spontaneously conceived pregnancy. Am J Obstet Gynecol 1997; 176: 1300-1302.

5. Haimov-Kochman R, Yanai N, Yagel S, Amsalem H, Lavy Y, Hurwitz A: Spontaneous ovarian hyperstimulation syndrome and hyperreactio luteinalis are entities in continuum. Ultrasound Obstet Gynecol 2004; 24: 675-678.

6. Bidus MA, Ries A, Magann EF, Martin JN: Markedly elevated beta-hCG levels in a normal singleton gestation with hyperreactio luteinalis. Obstet Gynecol 2002; 99: 958-961.

7. Schnorr JA, Miller H, Davis JR, Hatch K, Seeds J: Hyperreactio luteinalis associated with pregnancy: a case report and review of the literature. Am J Perinatol 1996; 13: 95-97.

8. Wajda KJ, Lucas JG, Marsh WL: Hyperreactio luteinalis: benign disorder masquerading as an ovarian neoplasm. Arch Pathol Lab Med 1989; 113: 921-925.

9. Quereda F, Acien P, Hernandez A: Hyperreactio luteinalis: intraoperative finding during a cesarean section in a twin pregnancy. Eur J Obstet Gynecol Reprod Biol 1996; 66: 71-73.

10. Bidus MA, Ries A, Magann EF, Martin JN: Markedly elevated beta-hCG levels in a normal singleton gestation with hyperreactio luteinalis. Obstet Gynecol 2002; 99: 958-961.

11. Smits G, Olatunbosun O, Delbaere A, Pierson R,
Vassart G, Costagliola S: Ovarian hyperstimulation syndrome due to a mutation in a follicle-stimulation hormone receptor. N Engl J Med 2003; 349: 760-766.

12. Onodera N, Kishi I, Tamaoka Y, Yamazaki K, Kamei $\mathrm{K}$ : A case of recurrent hyperreactio luteinalis. Am J Obstet Gynecol 2008; 198: e9-10.

13. Angioni S, Portoghese E, Milano F, Melis GB, Fulghesu AM: Hirsutism and hyperandrogenism associated with hyperreactio luteinalis in a singleton pregnancy: a case report. Gynecol Endocrinol 2007; 23: 248-251.

14. Gatongi DK, Madhvi G, Tydeman G, Hasan A: A case of hyperreactio luteinalis presenting with eclampsia. J Obstet Gynaeco 2006; 26: 465-467.

15. Gherman RB, Mestman JH, Satin AJ, Goodwin TM: Intractable hyperemesis gravidarum, transient hyperthyroidism and intrauterine growth restriction associated with hyperreactio luteinalis. A case report. J Reprod Med 2003; 48: 553-556.

16. Saisto T, Tiitinen A, Ulander VM, Kaaja R: Clinical cure of severe, early onset preeclampsia with low molecular weight heparin therapy in primigravida with hyperreactio luteinalis and thrombophilia. Hum Reprod 2004; 19: 725-728.

(Received, December 15, 2010)

(Accepted, March 2, 2011) 